\title{
Development of an Institution-Specific Readmission Risk Prediction Model for Real-time Prediction and Patient-Centered Interventions
}

J Gen Intern Med 36(12):3910-2

DOI: $10.1007 / \mathrm{s} 11606-020-06549-9$

(C) Society of General Internal Medicine 2021

\section{INTRODUCTION}

Barnes Jewish Hospital (BJH) has developed intervention programs for patients 65 and older diagnosed with acute myocardial infarction (AMI), congestive heart failure (CHF), chronic obstructive pulmonary disease (COPD), and pneumonia (PNA). Eligible patients are high-risk inpatients identified using the LACE Index Scoring Tool, ${ }^{1}$ which is the most widely used method to quantify the risk of readmission, but only achieves a C-statistic of 0.59 in our population, compared to range $0.63-0.70$ in other systems. ${ }^{2} \mathrm{Yu}$ et al. reported that "the institution specific readmission risk prediction framework is more flexible and more effective than the one-size-fit-all models like the LACE", ${ }^{3}$ and several groups have used machine learning models to improve readmissions predictions. ${ }^{4,5}$

Therefore, in this proof of concept study, we use available variables to better identify at-risk patients (compared to the LACE score) at BJH in order to enroll them into the current readmission reduction programs early in their hospital course. We developed a predictive model, which improved our predictions over the LACE score for real-time electronic medical record integration into the electronic medical record.

\section{METHODS}

The study cohort included 965 patients discharged from May 1, 2015, to April 30, 2016, with an index diagnosis of AMI, COPD, CHF, and PNA using discharge International Classification of Diseases 9 and 10 codes cross-walked. Figure 1 describes how the 776 unique patients were chosen and divided for model training and evaluation.

The primary outcome was readmission; variables available as predictors include total readmissions, diagnosis, diabetes comorbidity, average length of stay, age from 65 to $80+$ in 5 year groupings, race, primary care provider listed at the time of

Received August 25, 2020

Accepted December 22, 2020

Published online January 26, 2021 admission, discharge disposition, and LACE score by low, medium, or high designation.

We trained logistic regression, random forest, support vector machines, and AdaBoost models on the training set. Additionally, a model was developed using the TDA Mapper algorithm which divides the patients into sub-groups, and then trains independent models for each group. ${ }^{6}$ Model parameters are selected via cross-validation, and SMOTE/ROSE sampling was tested to address the low readmission rate.

Models were first trained on all available predictors. Then, models were trained excluding the LACE score and/or Discharge Disposition (DD). DD was excluded because it is not available early in the hospital stay.

\section{RESULTS}

The significant predictors $(p<0.001)$ with increased odds of readmission in the multivariate regression were LACE score (OR 1.22; 95\% CI 1.14-1.31); home health discharge (2.34;

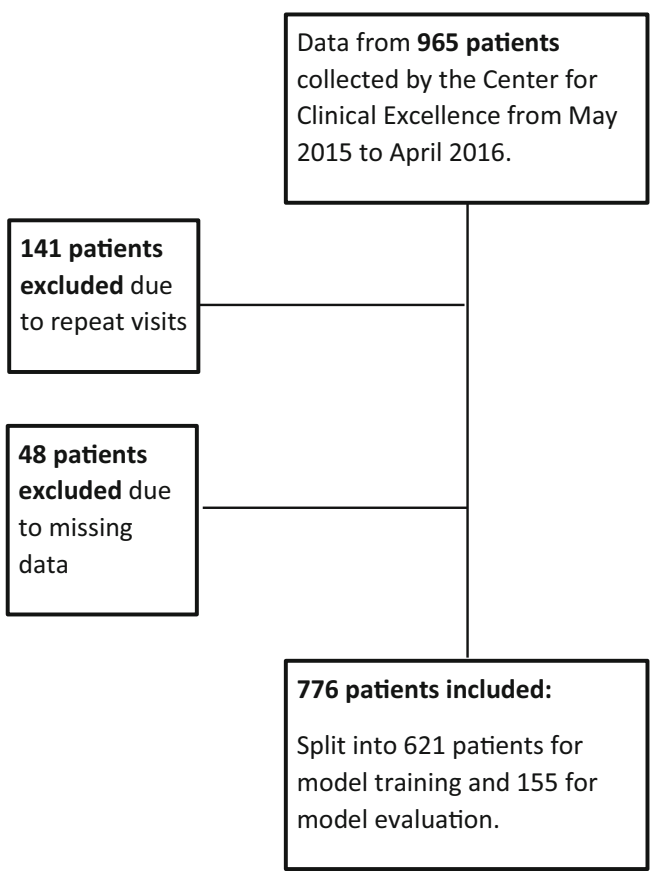

Figure 1 Information flow of unique patients analyzed. 
Table 1 Model Performance Comparisons with C-Statistic, Sensitivity, Specificity, and PPV, Averaged over $n=10$ Runs

\begin{tabular}{|c|c|c|c|c|}
\hline $\begin{array}{l}\text { Model } \\
\text { (model type, sampling, removed predictors) }\end{array}$ & C-Statistic & Sensitivity & Specificity & PPV \\
\hline BASELINE: univariate logistic regression, no sampling, only LACE & $0.59(0.55,0.63)$ & $0.54(0.49,0.59)$ & $0.58(0.54,0.63)$ & $0.21(0.17,0.25)$ \\
\hline Multivariate logistic regression, & $\begin{array}{l}0.49 \\
(0.44,0.54)\end{array}$ & & & $0.18(0.16,0.20)$ \\
\hline Multivariate logistic regression, SMOTE, All Data & $0.53(0.4$ & $0.67(0.60,0.73)$ & $0.44(0.37,0.51)$ & $0.20(0.18, .22)$ \\
\hline Multivariate logistic regression, SMOTE, No Disch. Disp. & $\begin{array}{l}0.54 \\
(0.5,0.58)\end{array}$ & $0.61(0.58,0.64)$ & $0.59(0.56,0.62)$ & $\begin{array}{l}0.224(0.19 \\
0.25)\end{array}$ \\
\hline Multivariate logistic regression, SMOTE, No LACE, No Disch. Disp & $\begin{array}{l}0.56 \\
(0.52,0.59)\end{array}$ & $0.67(0.63,0.73)$ & $0.55(0.52,0.58)$ & $0.24(0.21,0.27)$ \\
\hline Multivariate logistic regression with Mapper, SMOTE & $0.57(0.53,0.61)$ & $\mathbf{0 . 7 5}(0.72,0.78)$ & $0.46(0.43,0.49)$ & $0.22(0.18,0.26)$ \\
\hline Multivariate logistic regression with Mapper, ROSE, all data & $\begin{array}{l}0.63 \\
(0.62,0.64)\end{array}$ & $0.61(0.59,0.63)$ & $0.6(0.58,0.62)$ & $0.24(0.22,0.26)$ \\
\hline Random Forest with Map & $0.66(0.61,0.72)$ & $0.71(0.67,0.75)$ & $0.64(0.61,0.67)$ & $0.29(0.24,0.34)$ \\
\hline AdaBoost with Mapper, SMOTE, No Disch. Disp. & & & & \\
\hline $\begin{array}{l}\text { Multivariate logistic regression with Mapper, SMOTE No Disch. } \\
\text { Disp. }\end{array}$ & $\mathbf{0 . 6 9}(0.67,0.71)$ & $0.58(0.55,0.61)$ & $\mathbf{0 . 7 8}(0.74,0.82)$ & $0.35(0.33,0.37)$ \\
\hline
\end{tabular}

SMOTE, synthetic minority oversampling; ROSE, randomly oversampling; PPV, positive predictive value

Bolded numbers:Demonstrating that local prediction using machine learning tools without LACE can result in improved modeling

Model without the LACE score that balances the c-statistic, sensitivity, specificity and PPV the best was using Random Forest with Mapper

Model without LACE nor Discharge Disposition that performed best was the LR with SMOTE

1.57-3.49); male sex (1.97; 1.36-2.86); age 75-79 (1.78; 1.2 2.6); and having a primary care physician noted in the EMR (1.77; 1.5-2.72). Significant predictors with decreased odds were COPD $(0.21 ; 0.11-0.45)$ and AMI $(0.66 ; 0.45-0.99)$ (vs. CHF reference variable), discharge to a skilled nursing facility (0.50; 0.29-0.96), and age 70-74 (65-69 reference variable) $(0.69 ; 0.48-0.98)$.

Model performance was evaluated using the C-statistic, sensitivity, specificity, and positive predictive value, averaged over 10 different training-testing splits. We hypothesize that some of the false positives might also be at high risk for readmission and benefit from additional treatment options, and thus can accept a lower specificity in exchange for high sensitivity. The positive predictive value was reported although is skewed due to only $17 \%$ readmissions.

A model comparison is presented in Table 1. The best model in terms of C-statistic (0.69) and specificity (0.78) was the Mapper-based logistic regression. The best with respect to sensitivity $(0.75)$ was the multivariate logistic regression with Mapper, SMOTE, and No LACE. In general, Mapper-based models outperformed other models.

\section{DISCUSSION}

In our cohort, readmitted and non-readmitted patients are not statistically significantly different based on our available predictors. Furthermore, the LACE score is not a good predictor in our population. Our data is a small, demographically homogeneous group of traditional Medicare patients, and our models are limited by a low number of significant/available predictors and low readmissions rate. Within this challenging framework, we developed a model that outperforms the LACE score on our population.

We hypothesize that our model works because it captures multiple statistical processes underlying readmissions, while univariate/multivariate regression only captures one process. Further investigation is needed to determine differences between each group, as well as the optimal method to divide patients.

Future analyses will incorporate more predictor variables and a larger cohort to better discriminate between patients. Models created will aid in improved targeted interventions including the Stay Healthy Clinic and Stay Healthy Outpatient Program.

Acknowledgments: The authors thank the Department of Internal Medicine, as well as Melvin Blanchard, MD; Stanley Birge, MD; Luke Mathews; Maura Garascia, MSW, LCSW.

Ann-Marcia C. Tukpah, MD, MPH ${ }^{1}$

Eric Cawi, BSEE ${ }^{2}$

Laurie Wolf, $P h D, A S Q-C S S B B^{3}$

Arye Nehorai, $P h D^{2}$

Lenise Cummings-Vaughn, $\mathrm{MD}^{4}$

${ }^{1}$ Division of Pulmonary and Critical Care Medicine, Department of Medicine, Brigham and Women's Hospital,

Boston, MA, USA

${ }^{2}$ The Preston M. Green Department of Electrical and Systems Engineering, McKelvey School of Engineering, Washington University in Saint Louis,

Saint Louis, MO, USA

${ }^{3}$ Human Factors Implementation, Carilion Clinic, Roanoke, VA, USA

${ }^{4}$ Division of Geriatrics and Nutritional Science, Department of Internal Medicine, Washington University in Saint Louis,

Saint Louis, MO, USA

Corresponding Author: Lenise Cummings-Vaughn, MD; Division of Geriatrics and Nutritional Science, Department of Internal Medicine, Washington University in Saint Louis, Saint Louis, MO, USA (e-mail:lenise@wustl.edu). 
Funding Internal Medicine Mentors in Medicine Grant

\section{Compliance with Ethical Standards:}

This work was approved by the Washington University Institutional Review Board.

Conflict of Interest: Mr. Eric Cawi reports grants from the National Science Foundation during the conduct of this study. Dr. Arye Nehorai reports grants from ONR, I-Cares - Washington University in St. Louis (WUSTL), NIH, CTRFP - WUSTL, AFOSR, and NIH National Institute of Aging outside of the work of this study. The other authors have no conflicts of interest to disclose.

\section{REFERENCES}

1. van Walraven C, Dhalla IA, Bell C, Etchells E, Stiell IG, Zarnke K, et al. Derivation and validation of an index to predict early death or unplanned readmission after discharge from hospital to the community. Cmaj. 2010;182(6):551-7.
2. Low LL, Lee KH, Hock Ong ME, Wang S, Tan SY, Thumboo J, et al. Predicting 30-Day Readmissions: Performance of the LACE Index Compared with a Regression Model among General Medicine Patients in Singapore. Biomed Res Int. 2015;2015:169870.

3. Yu S, Farooq F, van Esbroeck A, Fung G, Anand V, Krishnapuram B. Predicting readmission risk with institution-specific prediction models. Artif Intell Med. 2015;65(2):89-96.

4. Futoma J, Morris J, Lucas J. A comparison of models for predicting early hospital readmissions. J Biomed Inform. 2015;56:229-38.

5. Garcia-Arce A, Rico F, Zayas-Castro J. Comparison of Machine Learning Algorithms for the prediction of Preventable Hospital Readmissions. Journal for Healthcare Quality, 2018; 40(3):129-138.

6. Cawi E, La Rosa PS, Nehorai. Designing machine learning workflows with an application to topological data anallysis. PLOS ONE. 2019. 14(12).

Publisher's Note: Springer Nature remains neutral with regard to jurisdictional claims in published maps and institutional affiliations. 